\title{
Transferência de lípides para a lipoproteína de alta densidade (HDL) em mulheres com diabetes melito tipo 1
}

\author{
Lipid transfer to HDL in women with type 1 diabetes
}

Alina Coutinho Rodrigues Feitosa', Raul Cavalcante Maranhão', Gilson Soares Feitosa Filho', Bernardo Léo Wajchenberg ${ }^{2}$

\begin{abstract}
RESUMO
Introdução: Os portadores de diabetes melito tipo 1 (DM1) possuem aumentado risco de doença cardiovascular e, ainda assim, podem apresentar perfil lipídico normal. Para esclarecer se os níveis normais de HDL podem ocultar defeitos na função, foram estudados a transferência de lípides para a HDL em DM1. Métodos: Vinte e uma mulheres jovens portadoras de DM1 foram comparadas com 21 mulheres não-diabéticas. Nanoemulsões foram usadas como doadoras de lípides para HDL: uma marcada com ${ }^{3} \mathrm{H}$-triglicérides $\mathrm{e}^{14} \mathrm{C}$-colesterol livre e outra com ${ }^{3} \mathrm{H}$-éster de colesterol e ${ }^{14} \mathrm{C}$-fosfolípides. Após 1 hora de incubação com amostras de plasma, seguida por precipitação química, o sobrenadante, contendo HDL, teve a radioatividade contada. Resultados: Nenhuma diferença foi encontrada nas transferências dos ésteres de colesterol, triglicérides, colesterol livre e fosfolípides para as HDL. Conclusão: A transferência de lípides para a HDL não está afetada em portadoras de DM1. Isso sugere que a doença não altera a composição de lipoproteínas e a ação de proteínas de transferência. Arq Bras Endocrinol Metab. 2009;53(1):95-101.
\end{abstract}

Descritores

Diabetes melito tipo 1; nanopartículas; colesterol HDL; lipoproteínas/metabolismo; controle glicêmico

\begin{abstract}
Introduction: People with type 1 diabetes mellitus (T1DM) have an increased risk of cardiovascular disease and may still have a normal lipid profile. In order to clarify whether normal HDL cholesterol levels may conceal defects in HDL function, we have studied the transfer of lipids to HDL in T1DM. Methods: Twenty-one young women with T1DM were compared with 21 non-diabetic women. Nanoemulsion preparations were used as lipid donor to HDL: one labeled with ${ }^{3} \mathrm{H}$-triglycerides and ${ }^{14} \mathrm{C}$-free cholesterol and the other with ${ }^{3} \mathrm{H}$-cholesteryl esters and ${ }^{14} \mathrm{C}$-phospholipids. These preparations were incubated with plasma samples for $1 \mathrm{~h}$. After chemical precipitation, the supernatant containing HDL was counted for radioactivity. Results: No difference in transfer was observed to nanoemulsion HDL from cholesteryl esters, triglycerides, free cholesterol and phospholipids. Conclusion: Simultaneous lipid transfer to HDL was not affected in T1DM patients. This suggests that the disease does not alter lipoprotein composition and transfer protein action in such way as to disturb HDL metabolism. Arq Bras Endocrinol Metab. 2009;53(1):95-101.
\end{abstract}

\author{
' Laboratório de Metabolismo \\ de Lípides do Instituto do \\ Coração (InCor), Hospital das \\ Clínicas, Faculdade de Medicina, \\ Universidade de São Paulo \\ (HC-FMUSP) \\ ${ }^{2}$ InCor, HC-FMUSP; São \\ Paulo, SP, Brasil.
}

Correspondência para:

Alina Coutinho Rodrigues Feitosa Coordenação de Ensino e Pesquisa do Hospital Santa Izabel

Praça Conselheiro Almeida Couto, 500 - Nazaré 40500-410 Salvador, BA alinafeitosa@yahoo.com.br

Recebido em 24/Jul/2008 Aceito em 17/Nov/2008

\section{INTRODUÇĀO}

$\mathrm{O}$ diabetes melito (DM) associa-se frequentemente a alterações nas lipoproteínas plasmáticas. A dislipidemia típica é caracterizada por hipertrigliceridemia, HDL baixo e LDL pequena e densa, entretanto, portadores de diabetes melito tipo 1 (DMl) apresentam-na em menor frequên- cia que os portadores de diabetes melito tipo 2 (DM2). A maioria dos DMl tratada intensivamente com insulina cursa com perfil lipídico normal (1), podendo ser encontradas concentrações reduzidas de LDL e elevadas de HDL (2-4). Ainda assim, as doenças cardiovasculares (DCV) são a principal causa de morte entre DMl. A concentração de HDL 
é inversamente associada ao desenvolvimento da aterosclerose (5), entretanto, em mulheres diabéticas, as DCV são a principal causa de morte (6), a despeito de apresentarem concentrações de HDL mais elevadas do que os homens (7). O impacto do diabetes no risco de morte por DCV na mulher é maior que o do homem (8).

Além da existência da relação inversa entre HDL e DCV, alterações no tamanho, na composição e na estrutura das HDL podem comprometer a funcionalidade e interferir com suas propriedades antiaterogênicas (9). A HDL está constantemente sendo remodelada e as transferências de lípides são essenciais para o transporte reverso e a esterificação do colesterol (10), importantes passos metabólicos na prevenção da aterosclerose. As transferências de lípides entre as classes de lipoproteínas são bidirecionais, mas, frequentemente, resultam depleção ou enriquecimento de dada classe de lípides. As transferências dependem das concentrações e da estrutura da lipoproteína doadora e aceptora (11), assim como da ação de proteínas de transferência (12) nomeadas proteína de transferência de ésteres de colesterol (CETP) (13) e proteína de transferência de fosfolípides (PLTP) (14). A insulina desempenha importante papel na regulação do metabolismo das $\operatorname{HDL}(15,16)$, atuando em proteínas de transferência (17) e regulando a produção das HDL (15). Considerando que a fisiopatologia do DMl é caracterizada por insulinodeficiência e que o tratamento é feito exclusivamente com insulina, alterações no metabolismo das HDL são necessariamente influenciadas pelo requerimento obrigatório deste hormônio.

Informações a respeito de transferência de lípides para as HDLs em DMl são escassas na literatura. Em pacientes DMl normolipidêmicos que recebem insulinoterapia subcutânea convencional, a transferência de éster de colesterol (CE) da LDL para a HDL foi semelhante a dos controles não-diabéticos, enquanto a transferência de triglicérides (TG) foi maior apenas na subfração $\mathrm{HDL}_{3}(18)$. Nesse estudo de Ritter e Bagdade, a técnica utilizada foi a de transferência de massa que avalia a composição lipídica da partícula HDL ao fim dos ensaios e estima a transferência a partir da depleção ou do enriquecimento das partículas. Ritter e Bagdade estudaram apenas dois lípides - TG e CE - e foi utilizado um inibidor da atividade da lecitina colesterol aciltransferase no plasma dos indivíduos, o que modifica parte da transferência, porquanto impede a esterificação do colesterol livre (CL).

A proteção antiaterogênica conferida pela HDL é mais importante no sexo feminino do que no sexo masculino, efeitos mediados por ações dos estrógenos $(19,20)$. Além disso, a mulher está mais exposta a DCV relacionada ao diabetes do que o homem (21). Dessa forma, é importante estudar, nessas pacientes, os vários aspectos do metabolismo da HDL, já que está demonstrado que a determinação da concentração plasmática da lipoproteína é insuficiente para avaliar o seu papel protetor. Assim, o presente estudo objetivou avaliar a atividade aceptora de lípides da HDL em pacientes DMl. Esse é um importante aspecto do metabolismo da lipoproteína, já que várias funções da HDL estão relacionadas com a transferência de lípides $(10,22)$. $\mathrm{Na}$ abordagem que foi utilizada, com fundamento em uma nanoemulsão artificial que funciona como doadora de lípides, pode quantificar-se, a um só tempo, a transferência de quatro lípides - CL, CE, TG e fosfolípides (FL). Além disso, foi avaliada a associação das transferências com o controle glicêmico e a insulinoterapia em esquema intensivo.

\section{MÉTODOS}

\section{PARTICIPANTES DO ESTUDO}

Vinte e uma mulheres portadoras de DMl e 21 controles não-diabéticas não parentes dos casos pareadas para idade, sexo e índice de massa corpórea (IMC) foram estudadas. Foram considerados critérios de exclusão: dislipidemia (LDL $>130, \mathrm{HDL}<40$ ou TG $>150 \mathrm{mg} / \mathrm{dL}$ ) ou uso de hipolipemiantes, câncer, doenças inflamatórias, uso de imunossupressores ou glicocorticóides, hipotireoidismo não controlado e complicações macro e microvasculares significativas. Essas foram definidas como: retinopatia proliferativa ou não-proliferativa moderada a grave, microalbuminúria acima de $50 \mathrm{mg}$ em 24 horas e neuropatia periférica caracterizada por alteração nos testes de rastreamento (teste do monofilamento de $10 \mathrm{~g}$ e teste da percepção da vibração alterado com diapasão $128 \mathrm{~Hz}$ ) ou eletroneuromiografia compatível, associados à história de lesão não percebida, ulceração ou amputação.

\section{EXAME CLÍNICO}

A revisão dos prontuários e a entrevista foram feitas para checar complicações, comorbidades e medicações de cada participante do estudo. Foram mensurados altura, peso, circunferência abdominal, relação cintura-quadril de todas as participantes. O IMC foi calculado dividindo-se o peso pela altura ao quadrado.

\section{ANÁLISES DA BIOQUÍMICA SANGUÍNEA}

Foram coletadas amostras de sangue após 12 horas de jejum para a determinação de lípides plasmáticos, apolipoproteínas, glicemia em jejum, hemoglobina glicada e 
para realização dos ensaios de transferências de lípides. Triglicérides, colesterol total (CT), HDL, VLDL e glicemia foram mensurados por meio do método enzimático colorimétrico automatizado e o colesterol de LDL pelo método cinético automatizado. As concentrações plasmáticas de apoAl e B foram determinadas pela turbidimetria (Roche/Hitachi - Roche Diagnostics - Mannheim), a hemoglobina glicada, pelo método HLPC, certificado pelo National Glyco Hemoglobin Standardization Program (NGSP, Estados Unidos), considerando a porcentagem entre $4,1 \%$ e $6,0 \%$ como valor normal.

\section{MENSURAÇÃO DO TAMANHO DA HDL}

O tamanho da partícula HDL foi medido pelo método do espalhamento de luz, segundo a técnica descrita por Lima e Maranhão (23).

\section{PREPARO DA NANOEMULSÃO LIPÍDICA PARA O ENSAIO DA CAPACIDADE ACEPTORA DAS HDL}

A nanoemulsão foi feita de acordo com o procedimento descrito por Ginsburg e Small (24) e modificado por Maranhão e cols. (25). A nanoemulsão foi preparada a partir da mistura lipídica composta de $40 \mathrm{mg}$ de oleato de colesterol, $20 \mathrm{mg}$ de fosfatildilcolina, $1 \mathrm{mg}$ de trioleína e 0,5 de colesterol adquirida da Sigma (Estados Unidos). Os lípides radioativos foram adquiridos da Amersham International (Amersham, UK) e foram adicionados às misturas lipídicas. Dois tipos de nanoemulsões foram preparadas, uma marcada com ${ }^{14} \mathrm{C}$-oleato de colesterol e ${ }^{3} \mathrm{H}$-colesterol e outra com ${ }^{14} \mathrm{C}$-fosfatidilcolina e ${ }^{3} \mathrm{H}$ trioleína. A emulsificação dos lípides por irradiação ultrassônica prolongada em meio aquoso e duas etapas de ultracentrifugação da emulsão com ajustes de densidade por meio da adição de $\mathrm{KBr}$ resultaram a nanoemulsão.

Para o ensaio da capacidade da HDL em receber os lípides radioativos da nanoemulsão, foram coletadas amostras dos pacientes, após jejum de 12 horas, em tubos contendo $0,15 \%$ de $\mathrm{Na}_{2}$ EDTA e o plasma foi obtido após 15 minutos de centrifugação, a $2.500 \mathrm{rpm}$ a $4^{\circ} \mathrm{C}$. Tubos teste contendo amostras de plasma $(0,2 \mathrm{~mL})$ e $0,05 \mathrm{~m}$ de nanoemulsão marcada com ${ }^{14} \mathrm{C}$-oleato de colesterol $\mathrm{e}^{3} \mathrm{H}$-colesterol ou com ${ }^{14} \mathrm{C}$-fosfatidilcolina e ${ }^{3} \mathrm{H}$-trioleína foram colocados em banho agitador e incubados por 60 minutos a $37^{\circ} \mathrm{C}$. Depois, $0,25 \mathrm{~mL}$ de solução contendo $0,02 \%$ de sulfato de dextran (50.000 MW) e $0,3 \mathrm{~mol} / \mathrm{L}$ de $\mathrm{MgCl}_{2}$ foi adicionada aos tubos e agitada em vórtex por 30 segundos. As amostras foram posteriormente centrifugadas por 10 minutos a $3.000 \mathrm{rpm}$ e $0,25 \mathrm{~mL}$ do sobrenadante conten- do a fração plasmática da HDL, obtidas e transferidas para frascos com solução cintiladora. A radioatividade presente nas amostras foi determinada em contador Beta (Liquid Scintillation Analyzer, Packard 1600 TR, Palo Alto, CA). A quantificação dos lípides transferidos da nanoemulsão para HDL plasmática foi calculada a partir do percentual de lípides marcados, doados da nanoemulsão e encontrados na fração da HDL plasmática.

O protocolo experimental foi aprovado pelo Comitê de Ética do Hospital das Clínicas da Faculdade de Medicina da Universidade de São Paulo (HC-FMUSP) e o termo de consentimento livre e esclarecido foi obtido de todos os participantes.

\section{ANÁLISE ESTATÍSTICA}

Todas as análises foram feitas com o SPSS v.13. Os resultados foram expressos em média e desvio-padrão para variáveis contínuas e valores absolutos e percentuais para variáveis categóricas. As variáveis contínuas foram comparadas conforme distribuição: se normal pelo teste t de Student e se não-normal pelo teste Mann-Whitney. As associações entre as variáveis contínuas foram avaliadas pelo testes de Pearson ou Spearman (paramétricas e não-paramétricas, respectivamente). Foram consideradas significativas as diferenças com $\mathrm{p}<0,05$, bicaudal.

\section{RESULTADOS}

\section{CARACTERÍSTICAS CLÍNICAS E LABORATORIAIS}

A Tabela 1 mostra dados clínicos e antropométricos, lípides, lipoproteínas plasmáticas, controle glicêmico e dose da insulina subcutânea. As diabéticas apresentavam longa duração da doença $(14,4 \pm 7,2$ anos) e controle glicêmico crônico ruim, com HbAlc de 8,8 \pm 1,3\%. O perfil lipídico, apolipoproteínas, circunferência abdominal, relação cintura-quadril e idade foram semelhantes. A maioria não fazia atividade física regular (sete [35\%] versus oito [38\%]), o uso de anticoncepcional foi semelhante entre DMl e controles (sete [32\%] versus seis [29\%]). Duas diabéticas apresentavam hipertensão $(9,5 \%)$, duas $(9,5 \%)$ microalbuminúria entre 30 e $50 \mathrm{mg}$ em 24 horas, três $(14,3 \%)$ retinopatia não-proliferativa leve, três $(15,8 \%)$ tinham alterações à pesquisa da vibração com diapasão de $128 \mathrm{~Hz}$, nenhuma apresentou alterações ao teste do monofilamento de $10 \mathrm{~g}$. A insulinoterapia em esquema intensivo ( 3 ou mais doses de insulina ao dia com ajustes de doses feitos com base em, pelo menos, três automonitorizações ao dia) era usada em $100 \%$ das DMl. A dose total diária de insulina foi de $0,67 \pm 0,17 \mathrm{UI} / \mathrm{kg}$. 
Tabela 1. Características antropométricas e clínicas, lípides e lipoproteínas plasmáticas, tamanho da partícula HDL e variáveis do metabolismo de carboidratos (média e desvio-padrão).

\begin{tabular}{|c|c|c|c|}
\hline Características & $\begin{array}{c}\text { Diabéticas } \\
(n=21)\end{array}$ & $\begin{array}{c}\text { Não- } \\
\text { diabéticas } \\
(\mathrm{n}=21)\end{array}$ & p \\
\hline Idade (anos) & $27,8 \pm 5,6$ & $30,8 \pm 6$ & 0,11 \\
\hline HAS (\%) & $2(9,5 \%)$ & 0 & 0,49 \\
\hline Atividade física* & $7(35 \%)$ & $8(38 \%)$ & 1 \\
\hline Uso de $\mathrm{IECA}^{\dagger}$ & $4(20 \%)$ & 0 & 0,048 \\
\hline Uso de ACO (\%) & $7(32 \%)$ & $6(29 \%)$ & 1 \\
\hline Tabagista & $5(24 \%)$ & $3(14 \%)$ & 0,67 \\
\hline $\mathrm{CA}(\mathrm{cm})$ & $85,5 \pm 9,4$ & $80,2 \pm 7,5$ & 0,07 \\
\hline $\mathrm{RCQ}$ & $0,75 \pm 0,05$ & $0,73 \pm 0,05$ & 0,21 \\
\hline IMC (kg/m²) & $24 \pm 3,7$ & $22,3 \pm 2,5$ & 0,18 \\
\hline apoA1 (mg/dL) & $158 \pm 34$ & $159 \pm 28$ & 0,88 \\
\hline apoB (mg/dL) & $75 \pm 17$ & $82 \pm 19$ & 0,25 \\
\hline $\mathrm{CT}(\mathrm{mg} / \mathrm{dL})$ & $174 \pm 34$ & $184 \pm 28$ & 0,28 \\
\hline LDLC (mg/dL) & $87 \pm 27$ & $96 \pm 27$ & 0,26 \\
\hline $\mathrm{HDLC}(\mathrm{mg} / \mathrm{dL})$ & $71 \pm 15$ & $69 \pm 16$ & 0,81 \\
\hline $\operatorname{VLDLC}(\mathrm{mg} / \mathrm{dL})^{\dagger}$ & $17 \pm 9$ & $19 \pm 7$ & 0,37 \\
\hline $\mathrm{TG}(\mathrm{mg} / \mathrm{dL})$ & $81 \pm 37$ & $93 \pm 34$ & 0,27 \\
\hline $\operatorname{HbA1c}(\%)^{\dagger}$ & $8,8 \pm 1,3$ & $5,3 \pm 0,4$ & $<0,001$ \\
\hline 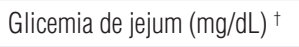 & $230 \pm 113$ & $71 \pm 8$ & $<0,001$ \\
\hline Dose/kg insulina (Ul/kg) & $0,67 \pm 0,17$ & - & - \\
\hline
\end{tabular}

${ }^{\dagger}$ Mann-Whitney ou teste exato de Fisher; * ${ }^{*}$ elo menos 3 vezes por semana em tempo superior a 30 minutos; HAS = hipertensão arterial sistêmica; IECA = inibidor de enzima conversora de angiotensina; $\mathrm{ACO}=$ anticoncepcional oral; $\mathrm{CA}=$ circunferência abdominal; $\mathrm{RCQ}=$ relação cintura-quadril; $I \mathrm{MC}$ = índice de massa corpórea; $a p o \mathrm{~A} 1=$ apolipoproteína $\mathrm{A} 1 ; \mathrm{apoB}=$ apolipoproteína $\mathrm{B} ; \mathrm{CT}=$ colesterol total; $\mathrm{LDLC}=\mathrm{LDL}$ colesterol; HDLC = HDL colesterol; VLDLC = VLDL colesterol; TG = triglicérides; HbA1c $=$ hemoglobina glicada A1c

\section{TAXAS DE TRANSFERÊNCIA DA NANOEMULSÃO PARA AS HDL E TAMANHO DA PARTÍCULA HDL}

As taxas de transferência de lípides das nanoemulsões para as HDL e o diâmetro médio da partícula HDL foram semelhantes entre diabéticas e controles (Tabela 2). As associações entre as taxas de transferências e o tamanho, lípides e apolipoproteínas plasmáticos e controle glicêmico estão na Tabela 3 .

Tabela 2. Taxas de transferência de lípides da nanoemulsão para as partículas HDL e tamanho (média e desvio-padrão).

\begin{tabular}{lccc}
\hline Dados & $\begin{array}{c}\text { Diabéticas } \\
(\mathbf{n}=\mathbf{2 1})\end{array}$ & $\begin{array}{c}\text { Não- } \\
\text { diabéticas } \\
\mathbf{( n = 2 1 )}\end{array}$ & $\mathbf{p}$ \\
\hline${ }^{14}$ C-éster de colesterol $(\%)$ & $3,9 \pm 1,3$ & $3,7 \pm 0,9$ & 0,63 \\
${ }^{14}$ C-fosfatidilcolina $(\%)$ & $21,5 \pm 3,6$ & $21,7 \pm 3,5$ & 0,83 \\
${ }^{3}$ H-triglicérides $(\%){ }^{\dagger}$ & $3,7 \pm 2,3$ & $3,6 \pm 1,3$ & 0,97 \\
${ }^{3} \mathrm{H}$-colesterol $(\%)$ & $6,8 \pm 1,6$ & $6,8 \pm 1,5$ & 0,87 \\
Tamanho da HDL $(\mathrm{nm})$ & $10,2 \pm 1,2$ & $9,7 \pm 0,9$ & 0,16 \\
\hline
\end{tabular}

Média $\pm \mathrm{DP} ;{ }^{\dagger}$ Mann-Whitney.
Tabela 3. Correlações entre as transferências de lípides da nanoemulsão para as $\mathrm{HDL}$ com diâmetro da partícula, controle glicêmico, dose de insulina, lípides, apolipoproteínas e IMC.

\begin{tabular}{|c|c|c|c|c|}
\hline & $\%$ TG & $\% \mathrm{CL}$ & $\% \mathrm{CE}$ & $\% \mathrm{PL}$ \\
\hline Tamanho da HDL (nm) & 0,15 & 0,10 & $-0,14$ & $-0,10$ \\
\hline 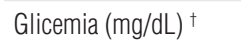 & $-0,19$ & $-0,21$ & $-0,18$ & 0,20 \\
\hline $\mathrm{HbA1C}(\%)^{\dagger}$ & 0,11 & 0,14 & 0,32 & 0,01 \\
\hline Insulina (UI/kg) & $-0,13$ & $-0,20$ & $-0,03$ & $-0,07$ \\
\hline apoA1 (mg/dL) & 0,28 & $0,56^{\star *}$ & $0,68^{* \star}$ & 0,25 \\
\hline $\mathrm{apoB}(\mathrm{mg} / \mathrm{dL})$ & 0,43 & 0,14 & 0,12 & $-0,021$ \\
\hline CT (mg/dL) & $0,47^{*}$ & 0,25 & 0,32 & 0,11 \\
\hline $\operatorname{LDLc}(\mathrm{mg} / \mathrm{dL})$ & 0,22 & $-0,025$ & $-0,09$ & $-0,11$ \\
\hline HDLC (mg/dL) & 0,21 & $0,53^{*}$ & $0,64^{\star \star}$ & 0,31 \\
\hline $\mathrm{TG}(\mathrm{mg} / \mathrm{dL})$ & 0,32 & 0,14 & 0,42 & 0,21 \\
\hline IMC $\left(\mathrm{kg} / \mathrm{m}^{2}\right)$ & 0,32 & $0,61^{\star *}$ & 0,25 & $-0,07$ \\
\hline
\end{tabular}

† apoA1 = apolipoproteína A1; apoB = apolipoproteína B; CT = colesterol total; LDLC = LDL colesterol; HDLC = HDL colesterol; VLDLC = VLDL colesterol; $\quad T G=$ triglicérides; $\mathrm{HbA1C}=$ hemoglobina glicada $\mathrm{A1C} ; \mathrm{IMC}=$ índice de massa corpórea. ${ }^{*}<0,05$; ** $<0,01$; † Spearman

\section{DISCUSSÃO}

As portadoras de diabetes avaliadas no estudo são jovens, normolipidêmicas, com mau controle glicêmico e não apresentam complicações significativas da doença. Em DMl sem complicações da doença e intensivamente tratados com insulina, a dislipidemia é incomum, acometendo cerca de $9 \%$ desta população (1).

O metabolismo de lípides no DMl é dependente da insulinopenia (26) e variável conforme o grau $(27,28)$ e forma de insulinização $(27,29-31)$.

$\mathrm{Na}$ ausência absoluta de insulina, a lipólise está aumentada, provendo o fígado de ácidos graxos livres que, associados à glicose elevada, fornecem o substrato para a formação das VLDL. Essas VLDL acumulam-se por causa da redução da atividade de lipase lipoprotéica (32), resultando a hipertrigliceridemia e a redução de HDL. A utilização de insulina para o tratamento origina espectro variável de alterações no metabolismo lipídico. A administração de insulina por via subcutânea resulta hiperinsulinismo que suprime a lipólise (33), ativa a lipase lipoprotéica (LLP) $(32,34)$ e reduz a glicemia, diminuindo o substrato para a formação de VLDL e aumentando a sua degradação via hidrólise mediada pela LLP. A insulina, ao ativar a PLTP (35) e a LLP, aumenta a HDL $(15,35)$.

A composição da HDL, que envolve lípides doados e recebidos entre lipoproteínas, é influenciada pela insulina $(28,30,36)$. A proteína de transferência CETP propicia a troca de ésteres de colesterol das HDL por TG com as partículas contendo apoB (13) e a PLTP medeia a transferência de FL e colesterol entre HDL e a 
interconversão das HDL (37). As ações destas proteínas alteram a composição e a estrutura da HDL. A literatura é controversa a respeito da atividade da CETP em DMI. A atividade da CETP pode estar aumentada (38), reduzida $(39,40)$ ou normal $(41,42)$, enquanto a PLTP está aumentada (43).

Em alguns trabalhos foi reportada a transferência no sentido da HDL para lipoproteínas contendo apoB no DMl $(18,28,31)$. Entretanto, até o momento, apenas um estudo avaliou a transferência no sentido das VLDL e LDL para a HDL, mostrando que não houve diferença na transferência de $\mathrm{CE}$ e TG para a fração $\mathrm{HDL}_{2}$ em comparação com os controles e transferência aumentada de TG apenas para a fração $\mathrm{HDL}_{3}(18)$. Neste estudo, as HDL foram depletadas em CE.

$\mathrm{Na}$ abordagem metodológica adotada no presente estudo, a nanoemulsão lipídica produzida artificialmente e que imita a composição da LDL foi utilizada como partícula doadora de lipídios para a HDL. Essa abordagem tem duas grandes vantagens: uma é a praticidade, já que se evitam os laboriosos procedimentos de marcação radioisotópica e isolamento do plasma das diversas lipoproteínas. Além disso, a nanoemulsão, em contato com o plasma, adquire as diversas apolipoproteínas plasmáticas e fica estruturalmente muito parecida com as lipoproteínas naturais. Usando-a como preparação-padrão única nos ensaios, evitam-se as variáveis inerentes às diferenças composicionais das lipoproteínas doadoras dos lípides, como as VLDL e as LDL, e assim o ensaio testa, especificamente, a capacidade da HDL do diabético de receber os lípides, independentemente das variações das lipoproteínas doadoras, como a glicação aumentada que ocorre no diabetes.

No presente estudo, não houve diferença na transferência de lípides da nanoemulsão para as HDL entre diabéticos e controles. A semelhança das transferências de lípides do estudo pode se dar na presença predominante da subfração $\mathrm{HDL}_{2}$ nos diabéticos, visto que, no grupo avaliado, a concentração da HDL estava elevada e o aumento da HDL nos DMl insulinizados ocorre em virtude do aumento da fração $\mathrm{HDL}_{2}(44)$.

Por meio do ensaio usando a nanoemulsão como doadora de lípides, a transferência é avaliada sob o ponto de vista unidirecional. Na direção oposta, o aumento de transferência de lípides da HDL para as lipoproteínas contendo apoB (36), com a doação de CE das HDL para as VLDL aumenta a proporção TG/CE nas HDL dos diabéticos (28). A depleção de CE no centro das partículas de HDL (18) diminui à concentração de HDL colesterol e apoAl e pode levar a alteração da estabilidade da HDL (45) e poderia prejudicar a função desta lipoproteína.
A semelhança do diâmetro das partículas HDL entre diabéticas e não-diabéticas encontrada neste estudo não permite afirmar que não haja diferenças de composição e função entre os dois grupos. O tamanho da partícula pode ser similar, porém com função vasodilatadora comprometida (46). Os portadores de DMl podem ter maior proporção de partículas grandes de HDL e menor de HDL de pequeno tamanho (47) e o aumento da quantidade de HDL de maior diâmetro pode ser explicado, em parte, pela ampliação da atividade da PLTP em diabéticos (43). Entretanto, a metodologia utilizada no presente estudo avalia a média do diâmetro de todas as partículas mensuradas, sem fracionamento de subclasses, e pela dispersão da luz poderia haver diferenças nos tamanhos das subclasses das HDL, que não foram avaliados.

O conjunto de resultados dos participantes deste estudo, no qual não houve diferenças não só do tamanho e dos parâmetros de transferência de lípides para a HDL, mas também na concentração plasmática do HDL colesterol, não obstante as restrições anteriormente formuladas, sugere-se que não haja, em mulheres jovens com esta doença, alterações acentuadas na composição e na ação das lipoproteínas de transferência. Caso contrário, isso seria refletido nos vários parâmetros aferidos neste estudo. Este resultado é diferente do que se encontra em pacientes com DM2 e em pacientes com síndrome metabólica (dados não publicados). Naqueles casos, em que o mecanismo fisiopatológico reside na resistência à insulina, houve alterações caracterizadas por aumento na transferência de lípides.

Apesar de a insulina influenciar as proteínas de transferência de lípides, não foram encontradas associações entre as taxas de transferências de lípides e as doses de insulina. A ausência desta associação pode ser resultado da absorção variável da insulina subcutânea para a circulação portal (48). A glicemia e a hemoglobina glicada não se associaram às transferências, mas as concentrações da apoAl e HDLc estiveram positivamente associadas à transferência de CL e CE. O aumento das taxas de transferência relacionado à elevação da concentração de HDLc sugere que tal achado resulte, predominantemente, da lei de ação de massas e não de aumento de atividade da CETP.

Uma questão importante é a de elucidar se ocorrem alterações na transferência de lípides para a HDL em pacientes com DMl que desenvolveram DCV. As pacientes que participaram deste estudo eram jovens, e a questão da presença de DCV remete à faixa etária mais elevada, tanto dos casos quanto dos controles. É possível que em idade mais elevada cenário diferente surja, em que a transferência esteja alterada e haja relação entre este fato e o desenvolvimento de complicações cardiovasculares. 
Em conclusão, em portadoras de DMl jovens, a capacidade da HDL em receber lípides não está alterada em comparação com controles não-diabéticas, mesmo em vigência de controle glicêmico não ideal. Em estudos futuros, seria interessante explorar os efeitos da idade e a relação com DCV sobre este mecanismo.

Agradecimentos: O presente estudo foi financiado pelo Conselho Nacional de Desenvolvimento Científico e Tecnológico (CNPq) e pela Fundação de Amparo à Pesquisa do Estado de São Paulo (Fapesp).

Este artigo é parte da tese de doutorado de Alina Coutinho Rodrigues Feitosa pelo Programa de Pós-graduação em Endocrinologia do Departamento de Clínica Médica do Hospital das Clínicas da Faculdade de Medicina da Universidade de São Paulo.

Declaração: Os autores declaram não haver conflito de interesses científicos pertinentes.

\section{REFERÊNCIAS}

1. Nathan DM, Cleary PA, Backlund JY, Genuth SM, Lachin JM, Orchard TJ, et al. Intensive diabetes treatment and cardiovascular disease in patients with type 1 diabetes. N Engl J Med. 2005;353:2643-53.

2. Winocour PH, Durrington PN, Ishola M, Anderson DC. Lipoprotein abnormalities in insulin-dependent diabetes mellitus. Lancet. 1986;1:1176-8.

3. Weis U, Turner B, Gibney J, Watts GF, Burke V, Shaw KM, et al. Longterm predictors of coronary artery disease and mortality in type 1 diabetes. QJM. 2001;94:623-30.

4. Kahri J, Groop PH, Viberti G, Elliott T, Taskinen MR. Regulation of apolipoprotein A-I-containing lipoproteins in IDDM. Diabetes. 1993; 42:1281-8.

5. Barter PJ, Rye KA. High-density lipoproteins and coronary heart disease. J Cardiovasc Risk. 1994;1:217-21.

6. Moss SE, Klein R, Klein BE. Cause-specific mortality in a populationbased study of diabetes. Am J Public Health. 1991;81:1158-62.

7. Idzior-Walus B, Mattock MB, Solnica B, Stevens L, Fuller JH. Factors associated with plasma lipids and lipoproteins in type 1 diabetes mellitus: the EURODIAB IDDM Complications Study. Diabet Med. 2001;18:786-96.

8. Lee WL, Cheung AM, Cape D, Zinman B. Impact of diabetes on coronary artery disease in women and men: a meta-analysis of prospective studies. Diabetes Care. 2000;23:962-8.

9. Sviridov D, Mukhamedova N, Remaley AT, Chin-Dusting J, Nestel P. Antiatherogenic functionality of high density lipoprotein: how much versus how good. J Atheroscler Thromb. 2008;15:52-62.

10. von Eckardstein A, Nofer JR, Assmann G. High density lipoproteins and arteriosclerosis. Role of cholesterol efflux and reverse cholesterol transport. Arterioscler Thromb Vasc Biol. 2001;21:13-27.

11. Ferretti G, Bacchetti T, Negre-Salvayre A, Salvayre R, Dousset N, Curatola G. Structural modifications of HDL and functional consequences. Atherosclerosis. 2006;184:1-7.

12. Albers JJ, Tollefson JH, Faust RA, Nishide T. Plasma cholesteryl ester and phospholipid transfer proteins and their regulation. Adv Exp Med Biol. 1988;243:213-7.

13. Tall AR. Plasma cholesteryl ester transfer protein. J Lipid Res. 1993;34:1255-74.

14. Tollefson JH, Ravnik S, Albers JJ. Isolation and characterization of a phospholipid transfer protein (LTP-II) from human plasma. J Lipid Res. 1988;29:1593-602.
15. Golay A, Zech L, Shi MZ, Jeng CY, Chiou YA, Reaven GM, et al. Role of insulin in regulation of high density lipoprotein metabolism. J Lipid Res. 1987;28:10-8.

16. Durrington PN. Serum high density lipoprotein cholesterol in diabetes mellitus: an analysis of factors which influence its concentration. Clin Chim Acta. 1980;104:11-23.

17. Taskinen MR. Lipoprotein lipase in diabetes. Diabetes Metab Rev. 1987;3:551-70.

18. Ritter MC, Bagdade JD. Changes in high density lipoprotein subfraction lipids during neutral lipid transfer in healthy subjects and in patients with insulin-dependent diabetes mellitus. Lipids. 1996;31:1-7.

19. Sumegova K, Blazicek P, Waczulikova I, Zitnanova I, Durackova Z. Activity of paraoxonase 1 (PON1) and its relationship to markers of lipoprotein oxidation in healthy Slovaks. Acta Biochim Pol. 2006;53:783-7.

20. Abplanalp W, Scheiber MD, Moon K, Kessel B, Liu JH, Subbiah MT. Evidence for the role of high density lipoproteins in mediating the antioxidant effect of estrogens. Eur J Endocrinol. 2000;142:79-83.

21. Howard BV, Cowan LD, Go O, Welty TK, Robbins DC, Lee ET. Adverse effects of diabetes on multiple cardiovascular disease risk factors in women. The Strong Heart Study. Diabetes Care. 1998;21:1258-65.

22. Tall AR, Jiang X, Luo Y, Silver D. 1999 George Lyman Duff memorial lecture: lipid transfer proteins, HDL metabolism, and atherogenesis. Arterioscler Thromb Vasc Biol. 2000;20:1185-8.

23. Lima ES, Maranhao RC. Rapid, simple laser-light-scattering method for HDL particle sizing in whole plasma. Clin Chem. 2004;50:1086-8.

24. Ginsburg GS, Small DM, Atkinson D. Microemulsions of phospholipids and cholesterol esters. Protein-free models of low density lipoprotein. J Biol Chem. 1982;257:8216-27.

25. Maranhão RC, Cesar TB, Pedroso-Mariani SR, Hirata MH, Mesquita $\mathrm{CH}$. Metabolic behavior in rats of a nonprotein microemulsion resembling low-density lipoprotein. Lipids. 1993;28:691-6.

26. Howard BV. Lipoprotein metabolism in diabetes mellitus. J Lipid Res. 1987;28:613-28.

27. Rosenstock J, Vega GL, Raskin P. Effect of intensive diabetes treatment on low-density lipoprotein apolipoprotein B kinetics in type I diabetes. Diabetes. 1988;37:393-7.

28. Bagdade JD, Dunn FL. Effects of insulin treatment on lipoprotein composition and function in patients with IDDM. Diabetes. 1992;41 Suppl 2:107-10.

29. Ruotolo G, Parlavecchia M, Taskinen MR, Galimberti G, Zoppo A, Le NA, et al. Normalization of lipoprotein composition by intraperitoneal insulin in IDDM. Role of increased hepatic lipase activity. Diabetes Care. 1994;17:6-12.

30. Bagdade JD, Dunn FL, Eckel RH, Ritter MC. Intraperitoneal insulin therapy corrects abnormalities in cholesteryl ester transfer and lipoprotein lipase activities in insulin-dependent diabetes mellitus. Arterioscler Thromb. 1994;14:1933-9.

31. Bagdade JD, Teuscher AU, Ritter MC, Eckel RH, Robertson RP. Alterations in cholesteryl ester transfer, lipoprotein lipase, and lipoprotein composition after combined pancreas-kidney transplantation. Diabetes. 1998;47:113-8.

32. Nikkila EA, Huttunen JK, Ehnholm C. Postheparin plasma lipoprotein lipase and hepatic lipase in diabetes mellitus. Relationship to plasma triglyceride metabolism. Diabetes. 1977;26:11-21.

33. Durrington PN, Newton RS, Weinstein DB, Steinberg D. Effects of insulin and glucose on very low density lipoprotein triglyceride secretion by cultured rat hepatocytes. J Clin Invest. 1982;70:63-73.

34. Taskinen MR, Nikkila EA, Nousiainen R, Gordin A. Lipoprotein lipase activity in adipose tissue and skeletal muscle of human diabetics during insulin deprivation and restoration. Scand J Clin Lab Invest. 1981;41:263-8. 
35. Huuskonen J, Olkkonen VM, Jauhiainen M, Ehnholm C. The impact of phospholipid transfer protein (PLTP) on HDL metabolism. Atherosclerosis. 2001;155:269-81.

36. Bagdade JD, Ritter MC, Subbaiah PV. Accelerated cholesteryl ester transfer in patients with insulin-dependent diabetes mellitus. Eur J Clin Invest. 1991;21:161-7.

37. Jiang XC, Zhou HW. Plasma lipid transfer proteins. Curr Opin Lipidol. 2006;17:302-8.

38. de Vries R, Kerstens MN, Sluiter WJ, Groen AK, van Tol A, Dullaart RP. Cellular cholesterol efflux to plasma from moderately hypercholesterolaemic type 1 diabetic patients is enhanced, and is unaffected by simvastatin treatment. Diabetologia. 2005;48:1105-13.

39. Hayashibe H, Asayama K, Nakane T, Kobayashi K, Amemiya S, Nakazawa S. Decreased activity of plasma cholesteryl ester transfer protein in children and adolescents with insulin-dependent diabetes mellitus. Acta Paediatr. 1999;88:1067-70.

40. Ohta T, Nishiyama S, Nakamura T, Saku K, Maung KK, Matsuda I. Predominance of large low density lipoprotein particles and lower fractional esterification rate of cholesterol in high density lipoprotein in children with insulin-dependent diabetes mellitus. Eur $\mathrm{J}$ Pediatr. 1998;157:276-81.

41. Valabhji J, Donovan J, McColl AJ, Schachter M, Richmond W, Elkeles RS. Rates of cholesterol esterification and esterified cholesterol net mass transfer between high-density lipoproteins and apolipoprotein Bcontaining lipoproteins in Type 1 diabetes. Diabet Med. 2002;19:424-8.
42. Medina WL, Nunes VS, Carrilho AJ, Shimabukuru AF, Lottenberg AM, Lottenberg SA, et al. High-density lipoprotein cholesterol esterification and transfer rates to lighter density lipoproteins mediated by cholesteryl ester transfer protein in the fasting and postprandial periods are not altered in type 1 diabetes mellitus. Eur J Intern Med. 2000;11:264-70.

43. Colhoun HM, Scheek LM, Rubens MB, Van Gent T, Underwood SR, Fuller $\mathrm{JH}$, et al. Lipid transfer protein activities in type 1 diabetic patients without renal failure and nondiabetic control subjects and their association with coronary artery calcification. Diabetes. 2001;50:652-9.

44. Mattock MB, Salter AM, Fuller JH, Omer T, Gohari RE, Redmond SD, et al. High density lipoprotein subfractions in insulin-dependent diabetic and normal subjects. Atherosclerosis. 1982;45:67-79.

45. Boucher J, Ramsamy TA, Braschi S, Sahoo D, Neville TA, Sparks DL. Apolipoprotein A-II regulates HDL stability and affects hepatic lipase association and activity. J Lipid Res. 2004;45:849-58.

46. Persegol L, Foissac M, Lagrost L, Athias A, Gambert P, Verges B, et al. $\mathrm{HDL}$ particles from type 1 diabetic patients are unable to reverse the inhibitory effect of oxidised LDL on endothelium-dependent vasorelaxation. Diabetologia. 2007;50:2384-7.

47. Colhoun HM, Otvos JD, Rubens MB, Taskinen MR, Underwood SR, Fuller JH. Lipoprotein subclasses and particle sizes and their relationship with coronary artery calcification in men and women with and without type 1 diabetes. Diabetes. 2002;51:1949-56.

48. Binder C, Lauritzen T, Faber O, Pramming S. Insulin pharmacokinetics. Diabetes Care. 1984;7:188-99. 